\title{
Interest of the Rapid Diagnostic Test in the Management of Streptotoccic Angina in Children
}

\author{
Maryam Mouamin ${ }^{1, *}$, Fadl Mrabih Rabou Maoulainaine ${ }^{2}$, Nabila Soraa ${ }^{1}$ \\ ${ }^{1}$ Microbiology Laboratory of Marrakech Mohamed VI University Hospital Center, Faculty of Medicine and Pharmacy, Qadi Iyyad \\ University, Marrakech, Morocco \\ ${ }^{2}$ Neonatology Department of Marrakech Mohamed VI University Hospital Center, Faculty of Medicine and Pharmacy, Qadi Iyyad \\ University, Marrakech, Morocco
}

Email address:

m.maryam2790@gmail.com (M. Mouamin)

${ }^{*}$ Corresponding author

\section{To cite this article:}

Maryam Mouamin, Fadl Mrabih Rabou Maoulainaine, Nabila Soraa. Interest of the Rapid Diagnostic Test in the Management of Streptotoccic Angina in Children. International Journal of Infectious Diseases and Therapy. Vol. 5, No. 1, 2020, pp. 9-16.

doi: $10.11648 /$ j.jijidt.20200501.13

Received: January 21, 2020; Accepted: February 11, 2020; Published: February 21, 2020

\begin{abstract}
The objective of this work was to evaluate the interest of RDT compared to the scores of clinical presumptions (McIsaac, PCIE, WHO) in the management of angina with SBHGA of the child while referring to the bacteriological culture. We have managed a prospective analytical study carried out over 8 months between July 2012 and February 2013 in the pediatric emergency department of the Mohammed VI UHC in Marrakech in collaboration with four pediatric practices in the liberal sector, in partnership with a Swiss medical research team from the Lausanne childhood Hospital. For each child consulting for acute angina, a clinical information sheet was completed, an RDT was carried out by the examining doctor, then a throat sample used for the classic bacteriological examination was carried out and treated by the microbiology laboratory of the Mohamed VI UHC of Marrakech. 124 children with strong suspicion of strep throat were included in the study. The average age was 6 years and 4 months with a sex ratio of 1.4 in favor of boys. The bacteriological culture demonstrated the group A hemolytic $\beta$ streptococcus in=45 (36,9\%). The analysis of the established clinical scores revealed the a very good sensitivity reaching $100 \%$ but with a mediocre specificity not exceeding 7,7\% any score combined without notable superiority of one compared to the others. Mc Isaac's score was more positive $\geq 4$ since RDT and culture were positive. The RDT showed a sensitivity of $62,2 \%$ and a specificity of $96,15 \%$ compared to the bacterial culture which is the reference examination. We retained a strong resistance to erythromycin reaching $69 \%$ associated with co-resistance to lyncomicin and spiramycinin $30 \%$ of cases. The bacterial origin to SBHG A acute angina in children from the city of Marrakech represented a third of the cases. RDT, by its sensitivity, its high specificity, its speed and its reproducibility, offers a very good means of diagnostic and therapeutic orientation and allows initiating early targeted antibiotic treatment to avoid the appearance of complications in the short, medium and long term and remedy the alarming situation of resistance to macrolides in Marrakech.
\end{abstract}

Keywords: Acute angina, Group A Beta-hemolytic Streptococcus, Clinical Scores, Rapid Diagnostic Test (RDT), Macrolide Resistance

\section{Introduction}

Acute tonsillitis is one of the leading causes of pediatric consultation and it is estimated that several million prescriptions are written every day around the world to treat them. Each year, 9 million tonsillitis are treated in France [1]. In the majority of cases, in $60 \%$ to $90 \%$, they are of viral origin and do not require antibiotic treatment [2]; either they are essentially an etiological problem, namely their viral or bacterial origin. The most feared germ, because of its frequency and possible complications, is group A betahemolytic streptococcus (GABHS) which is implicated in $25 \%$ to $40 \%$ of angina in children [2, 3].

Diagnostic tools; clinical such as the Mc Isaac score, the WHO criteria for the treatment of strep throat described in 
the strategy for integrated management of the child (IBD) or biological, namely rapid diagnostic tests (RDT) of the betahemolytic group A streptococcus, improve the etiological distinction between viral and bacterial angina in everyday practice and condition the therapeutic attitude. The objective of our work is to highlight the interest of RDT compared to clinical scores and its reliability compared to conventional culture techniques in the demonstration of GABHS for a more rational antibiotic therapy.

\section{Patient and Method}

This is a prospective analytical study carried out over 8 months between July 2012 and February 2013 in the pediatric emergency department of the Mohammed VI UHC in Marrakech with the collaboration of four pediatric practices in the liberal sector, in partnership with a medical team from Swiss research from the pediatric emergency department of the Lausanne Children's Hospital which provided us with rapid diagnostic tests for angina. This study collected a sample of 124 children aged 1 to 15 years consulting for an oropharyngeal infection while excluding, subsequently, 5 children aged $<3$ years to comply with the literature and who were initially included given the flagrancy of their clinical symptomatology. This sample was taken after recruiting and examining more than 1,000 children to have sufficient confidence intervals around the values studied in the extreme score groups, which were numerically smaller than the intermediate score groups. Were included in this study all the children from 3 to 15 years old coming in consultation for an oropharyngeal infection with strong suspicion of angina defined by the presence of signs of inflammation of the pharynx and/or tonsils during the examination clinical.

Parent's consent was obtained in writing before hand. We excluded children on antibiotic therapy or having taken it in the 7 days preceding the consultation.

The RDT kit used was the Strep A Quick Vue In-Line test from QUIDEL laboratories represented in Switzerland by the firm BioMérieux. This test has a sensitivity of $92 \%$, a specificity of $99 \%$ if one scrupulously respects the various stages of its progress.

Patient data were collected on an anamnestic, clinical and biological information sheet. 2 samples were taken by swabbing tonsils, one of which was intended for carrying out the RDT during the consultation and the other sent to the microbiology laboratory of the Mohammed VI UHC in Marrakech for cultivation. The inoculation was carried out on blood agar, anaerobically, with subculturing of the betahemolytic colonies for final identification.

The study of the sensitivity to erythromycin, lincomycin and spiramycin was made by antibiogram on agar media according to there commendations of the CA-SFM. The data were analyzed using SPSS20.0 statistical software.

\section{Results}

During the period of our study, 124 children with an oropharyngeal infection with strong suspicion of acute angina, consulting the pediatric emergency department of UHC Mohammed VI or in private settings, were examined and benefited from a throat swab which used to carry out a rapid diagnostic test for group A beta-hemolytic streptococcus (GABHS). The majority of children included in the study came from families of low socio-economic status.

The average age of our patients was 6 years and 4 months with a sex ratio of 1.4 in favor of boys. During the study period we noted that angina, in the population studied, occurred mainly in summer in July and August representing $42 \%$ of all patients (figure 1). GABHS was identified in $36.9 \%$ of the cultures. The RDT was positive in $28.2 \%$ (Figure 2). However, $20.3 \%$ of the cultures were positive for other pathogens.

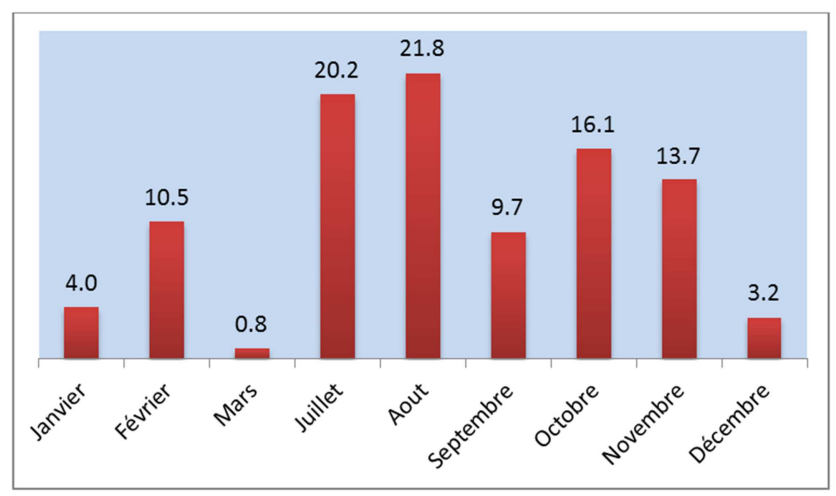

Figure 1. Distribution according to months of consultation.

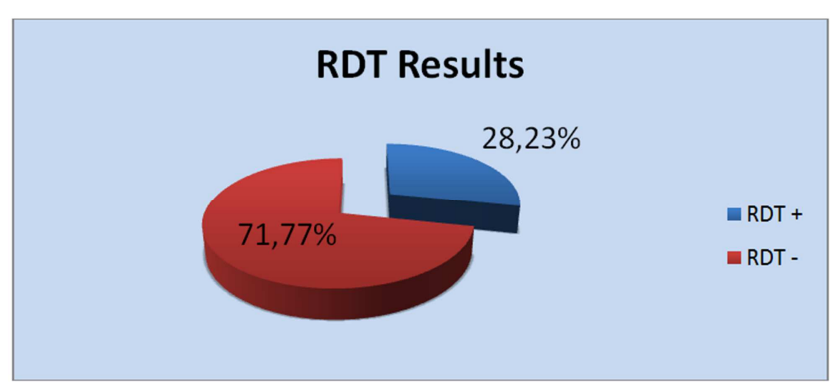

Figure 2. RDT results.

The clinical scores showed a high level of positivity with $100 \%$ of cases positive for Mc Isaac and considering the positivity threshold $\geq 1$, the PCIE was also positive in $96.8 \%$ and all ages combined. On the other hand, we retained a very poor correlation between the clinical scores assuming the streptococcal origin of angina and the RDT + not exceeding $30 \%$ for the different age groups and without superiority of a score clinch compared to others. However, we found an excellent correlation between the RDT + and cultures + which was $83.3 \%$ for experienced children from 3 to 5 years old and $76.9 \%$ for those who were from 5 to 15 years old, which testify to the good sensitivity of RDT.

Considering the culture as a reference test, the sensitivity was $62.2 \%$ and the specificity $96.15 \%$. The positive predictive value (VPP) was $96.5 \%$ and the negative 
predictive value (VPN) was $59.5 \%$. The clinical presumption scores presented an excellent sensitivity of $100 \%$, but a very low specificity not exceeding $7.7 \%$ for PCIE and WHO and $0 \%$ for Mc Issac. Of the 35 cases of RDT + , we only had 7 false positives (i.e. unnecessary antibiotic therapy). However, of the 89 cases of RDT- we found 16 false negatives with cultures positive for GABHS.

We have studied the sensitivity of SBHGA strains to macrolides. $66 \%$ of the isolated SBHGA strains retained their sensitivity to lincomycin and streptomycin. However, we found a high resistance level reaching $69.3 \%$ for erythromycin (Figure 3).

\section{Sensibility profil of GABHS}

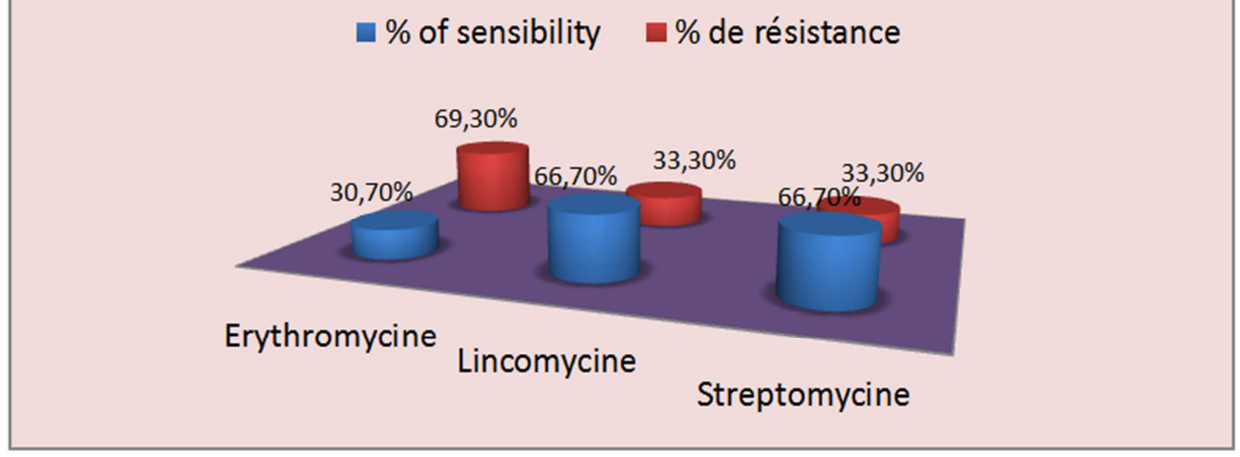

Figure 3. Sensitivity profile of GABHS to macrolides.

\section{Discussion}

Angina is defined by the French-language infectious pathologies society (SPILF)" as an inflammation of infectious origin of the tonsils or even of the entire pharynx". In children, it is viral in 60 to $90 \%$ [1], and bacterial in 20 to $40 \%$ with the group A hemolytic bet a streptococcus at the rate of 25 to $40 \%$. Note that bacterial super infection of viral angina is not uncommon. Angina with SBHGA can occur from the age of 3 years; its peak of incidence is located in children between 5 and 15 years old [1].

Other bacteria, in particular other beta-hemolytic streptococci (notably C, Fand G) maybe involved, but more rarely; they do not share the risk of rheumatic fever (RAA) of group A hemolytic $\beta$ streptococcus [1].

Given the low specificity of the clinical signs of angina with SBHG A causing prescriptions of antibiotics by excess, means of helping the diagnosis of these angina were developed in the 1980s. These are clinical scores.

The first established was the Breese score, [2] but took in to accounthy per leukocytosis, making it an impractical clinical and biological score.

In the $1980 \mathrm{~s}$, the Cent or score [3], which takes in to account 4 clinical criteria, was developed. The probability of finding the SBHGA when 4 criteria were present was $56 \%$, it fell to $30-34 \%$ when only 3 criteria were present, fell to $6 \%$ when only one criterion was present, and finally fell to $2.5 \%$ when they were all away [3]. However, this diagnostic method based only on 4 criteria has not been evaluated in children.

In 1998, using the Centor score, Mc Isaac [4] sought to improve the sensitivity and specificity of this score. He developed a score from clinical signs significantly associated with an SGA positive culture in 521 patients with pharyngitis and took into account the patient's age. The sensitivity was then assessed at $85 \%$ and the specificity at $92.1 \%$ [5].

The Mc Isaac score reinforces the clinical presumption of the streptococcal origin of angina in a population where the usual prevalence is around $15 \%$. It directs towards the realization of an RDT from a result $\geq 2$. It shows a good sensitivity in children but weak in adults, while its specificity is good in adults but weak in children [5]. This score therefore gives a clinical indication but does not allow a reliable diagnosis of angina with SBHGA to be made.

We also cite the WHO clinical score, applied in Egypt in children from 5 to 12 years old which reveals a specificity of 93.8 to $97.4 \%$, but a very low sensitivity of 3.6 to $8.5 \%$. This is unsatisfactory in management based solely on the clinic, given the high percentage of false negatives. [6]

Each year in developing countries nearly 12 million children die before reaching their 5 birthdays and many of them before the age of one. More than $70 \%$ are due to acute respiratory infections, diarrhea, measles, malaria or malnutrition [7]. Thus, the responsible authorities have developed a strategy to ensure better management of childhood illnesses, certain aspects of nutrition, vaccination and various other factors important for the child's health:

The Integrated Management of Childhood Illness (IMCI) strategy has three main components [7]: integrated care for sick children in health facilities and centers, strengthening of health systems, including drug supply and logistical support, and community IMCI or promotion of key family and community behaviors.

In Morocco, in 2002, a practical guide was published by the Ministry of Health to improve the management of angina based on the clinic at basic health facilities. Antibiotic therapy is only indicated if symptoms persist beyond 3 days [8]. 
In 2012, the Moroccan Ministry of Health adopted the IMCI of WHO, and issued an algorithm intended for all pediatricians in Morocco based on clinical criteria in order to distinguish between strep throat or not for better management of antibiotics intended to treat child's angina [9].

The diagnostic strategy chosen must be sensitive enough since patients with a false negative result may develop complications, and it must be specific enough since false positive results may lead to the consumption of unnecessary antibiotics.

On an oropharyngeal sample two techniques can confirm the presence of SGA: the practice of a rapid diagnostic test (RDT) and the culture of the sample, the latter remains the reference technique, although the proportion of false negative estimates varies from 1 to $10 \%$ [10]. It is a classic culture technique on blood agar, without inhibitor, incubated 24 or better 48 hours in ambient air with a sensitivity and specificity of 90 to $95 \%$. However in practice, cultures are poorly performed and are not recommended apart from rare indications including the search for resistance to macrolides and ketolides on the antibiogram data. Whichever technique is used, the result is obtained within 1 to 2 days, which is not efficient enough in the area of angina.

In 2002, in its recommendations for good practice in the presence of erythematous or erythematato-pultacea tonsillitis, AFSSAPS (French Agency for Sanitary Safety of Health Products) adopted the treatment strategy based on the use of RDTs, as an alternative to classical bacteriology (culture on agar) which is practically impossible on a large scale [6].

The principle of these tests consists, starting from an oropharyngeal sample by swab, to demonstrate the polysaccharide antigen of streptococcus A by an antigenantibody reaction in the presence of specific antibodies. Its completion time is estimated at 10 minutes. Its sensitivity is more than $90 \%$ with a specificity close to $95 \%$ [6].

The RDT is recommended in any patient with erythematous or erythematato-pultacea angina: a positive test, confirming the etiology with $\beta$-hemolytic streptococcus A, justifies the prescription of antibiotics, and a negative test in a subject without risk factor rheumatic fever (RAA) does not justify systematic additional control by culture, nor antibiotic treatment. Only analgesic and antipyretic treatments are then useful [6].

Culture is only indicated if the RDT is negative and there are risk factors for RAA [6].

These situations evoking a context at risk for RAA are the personal history of RAA, the age between 5 and 25 years associated with a history of multiple episodes of angina with SGA or the concept of stays in regions endemic for RAA (Africa, French overseas departments and territories) and possibly certain environmental factors (social, health and economic conditions, overcrowding, closed community) [6].

However, confirming any negative RDT with a throat swab culture would be an overly costly attitude.

In Morocco the incidence of RAA remains high with more than 7000 cases treated per year according to data from the Ministry of Public Health. [11]
RAA is a systemic, non-suppurative inflammatory pathology occurring 15 to 20 days after an upper airway infection with group A hemolytic beta streptococcus. It must follow angina in people predisposed to certain strains of pyogenic Streptococcus [12, 13]. It is an autoimmune disorder and the main targets, joint and heart, are considered to result from a structural analogy between SBHGA and these tissues. The type of Streptococcus protein M plays an important role in the determinism of reactions.

One of the most feared complications of post-streptococcal syndrome is also acute post-streptococcal glomerulonephritis (ANG), which may have a cutaneous or pharyngeal starting point. The nephritogenic strains of streptococcus are most often distinct from rheumatogenic strains [14]. A study carried out in Scotland in 1976-79 in children 0-13 years old, estimated the annual incidence of GNA at $2.1 / 100,000$; the estimated risk is little different after SGA angina whether it is treated or not; pharyngeal prodromes are noted in half of the cases [14].

Prescribing antibiotics in SGA tonsillitis has several objectives [15]: To speed up the disappearance of symptoms, even if spontaneous healing of symptoms is the rule, their duration is reduced by about 24 hours with antibiotics provided " be prescribed early; reduce the spread of SGA to those around them since patients are no longer contagious 24 hours after the start of antibiotic treatment [16]. Without treatment, eradication of SGA can be achieved in longer periods of time, up to 4 months [17]. The reduction in pharyngeal carriage by antibiotics results in the negativation of SGA cultures in at least $90 \%$ of patients at the end of a correctly conducted treatment [18]; and lastly to prevent nonsuppurative post-streptococcal complications, notably RAA.

The only controlled studies that have established the preventive power (primary and secondary prevention) of antibiotics on RAA have been carried out with penicillin $G$ injectable in angina [19]. In patients with SGA (cultureverified) angina treated with intramuscular penicillin $G$, the RAA level is reduced by approximately $25 \%$. [20]

There is no study showing that treatment with oral penicillin $\mathrm{V}$ for 10 days also effectively prevents the occurrence of RAA, but the rate of eradication of pharyngeal SGA is achieved in proportions comparable to injectable penicillin. There is also no study demonstrating that antibiotic treatment with amoxicillin, macrolides, certain cephalosporins, have the same preventive power vis-à-vis RAA but they have proven their effectiveness on the eradication of SBHGA of the pharynx.

The treatment of strep throat with penicillin, used in different forms, was proposed by the American Heart Association Council 40 years ago, following the work of Denny and Wannamaker $[21,22]$. Penicillin V, which is more manageable than the injectable forms, has become a priority in this treatment, which has only recently been confirmed [23-25]. In the event of intolerance to $\beta$-lactams, use is made of old macrolides (erythromycin, josamycin) at a dose of 40 to $50 \mathrm{mg} / \mathrm{kg}$ also for 10 days, or even more recently azithromycin, of which 1 activity in 5 days is recognized by 
the AMM. These recommendations, universally applied and official in France, have made it possible to eradicate RAA, at least in countries with a high standard of living, provided that treatment is initiated, at the latest before the 7 th day of the development of angina and imperatively followed for 10 days to allow the maintenance at the amygdala of an effective antibiotic concentration (higher than the MIC of the SGA) [26, 27].

In the early $1970 \mathrm{~s}$, new therapeutic methods were introduced because they demonstrated better efficacy compared to Peni $\mathrm{V}$, in particular $\mathrm{C} 1 \mathrm{G}$, but with a treatment duration always of 10 days.

Amoxicillin had also demonstrated very good bacterial eradication superior to that of penicillin $\mathrm{V}$, according to a study by Shvartzman [28] on 157 patients, in a daily dose of $50 \mathrm{mg} / \mathrm{kg}$ for 10 days. This duration was subsequently reduced to 6 days under $50 \mathrm{mg} / \mathrm{kg}$ per day divided into 2 times and this according to a study by Cohen [27].

Currently penicillin by injection has been abandoned especially by pediatricians due to its inconvenience and pain inflicted on children, but retains its place in case of noncompliance with prolonged oral treatment and in case of a high risk of RAA or relapses with a single intramuscular injection [6].

The evolution of angina under antibiotic treatment is most often favorable. The persistence of symptoms after three days of treatment should lead to re-examining the patient.

Symptomatic treatment meanwhile aims to improve patient comfort. It is used either alone as a supportive treatment in viral angina, or in combination with antibiotic therapy in the treatment of bacterial angina.

Pharyngitis occurs $95 \%$ in developing countries. In the United States, about 10 million children visit a pediatrician or general practitioner each year for pharyngitis, and $60 \%$ to $70 \%$ of these consultations result in a prescription for antibiotics [29, 30]. Similar rates of prescription of antibiotics are observed in France [31]. No prospective epidemiological study has been carried out in Morocco, therefore we are not aware of the annual consultation rates for pharyngitis in pediatrics, nor of the importance of prescribing antibiotics in Morocco.

Overuse of antibiotics is the main factor behind the emergence of antibiotic resistant bacteria and the majority of human consumption of antibiotics takes place outside the hospital (community consumption). [32]

In practice, and because of the possible risks of angina to SBHGA (in particular of RAA considering that we are an endemic country) and because of the difficulties of the diagnosis of their streptococcal origin in current practice, the Moroccan ministry of health recommends that all angina is treated with antibiotics [33]. This attitude leads to treating a very large number of angina, while the benefit of antibiotics has not been proven in non-strep throat.

Our population was predominantly male with $53.2 \%$, that is, a sex ratio of 1.14 in favor of boys with an average age (standard deviation) of 6.37 (2.96) years. A male predominance with $55.3 \%$ has also been reported by a French study carried out in 2014 [34] with an average age of 6.1 years. In another study done in the pediatric emergency department of Flaubert Le Havre Hospital in France, the average age was 4.8 plus or minus 3.6 years [35]. Nationally, we find a predominance of women with a sex ratio of 1.2 and an average age of $7 \pm 3$ years in a study carried out in Rabat [36].

Angina was diagnosed with a predominance in summer and fall, ie $81.5 \%$ isolated between July and November with a peak in August and a second in October, unlike other studies where the prevalence was mainly winter and spring [35]. In Sfax in Tunisia, the monthly distribution of sore throat shows a first peak at the start of the summer season in July and a second peak during the autumn period between October and November [37], results which approximate ours. But conventionally, strep throat is more common in winter and early spring [38-40].

The SBHGA was isolated from 45 cultures, ie $36.2 \%$ of all samples taken, fairly close proportions have been reported by other studies, notably at pediatric emergencies in Le Havre in France with 26\% [35], 36, 3\% in France also during the 2014 work [34] or in Sfax in Tunisia with $32.9 \%$ [37] of positive culture at SBHGA. A much lower rate was exceptionally found in Rabat with 9.1\% of isolated SBHGA [36].

Mac Isaac's score was all the more positive $\geq 4$, as the RDT was positive and the culture positive for SBHGA, something demonstrated by other studies, notably a Tunisian study in 2010 [36]. Also, the 2014 French study reported that $41.2 \%$ of children in whom SBHGA had been isolated had a Mac Isaac score $\geq 4$ [34].

RDTs generally had excellent specificity, often exceeding $95 \%$, and poorer sensitivity between 80 and 95\%. [41, 42] In our case, the RDT was positive in $28.23 \%$ of the cases and had a sensitivity of $62.22 \%$ and a specificity of $96.15 \%$ compared to the culture as a reference examination, $33.9 \%$ of positivity was also noted in the Tunisian study with $93.2 \%$ sensitivity and a specificity of $95.3 \%$ [36], which also corresponded to other data in the literature.

The positive predictive value (VPP) was $96.55 \%$ and the negative predictive value (VPN) $59.52 \%$, which was close to the results of pediatric emergencies in Le Havre in France with a UVPP at $60 \%$ and a VPN at $91 \%$ [35].

Note that the results of the RDT are a function of different factors, namely the size of the bacterial inoculum which mainly influences the sensitivity, the quality of the sample and the experience of the sampler (a good quality sample requires a swab of the 2 tonsils) and the patient's age [43].

A negative test therefore does not exclude strep throat. In our case we had $3.45 \%$ false positive rate with $19 \%$ false negative. For this reason the IDSA (Infectious deseaces Society of America) and the American Academy of Pediatrics [44] recommend that children confirm a negative RDT with a throat culture. This attitude involves an excessive cost [45]. In order to assess its impact on the outcome of patients, Webb et al. prospectively compared 2 groups of patients between 1997 and 1999 [46]. The difference between these 2 groups was whether or not a 
culture-based negative RDT was confirmed. The number of local complications was identical in the 2 groups. But in this study, the sensitivity was relatively high $(81.7 \%)$ and it was not a population of children more at risk for strep throat [46].

The use of RDT in a pediatric emergency unit has a very interesting impact on the prescription of antibiotics. During our study, the use of RDT made it possible to reduce the prescription of antibiotics by $70 \%$, since it was reserved for patients with SBHGA, there were added thereafter the cases of false negatives estimated at $13 \%$ that were recalled after culture results; a saving of more than $50 \%$ of antibiotics. This saving was $80 \%$ for other studies [35]. Previous data show that using RDT results in fewer prescriptions for antibiotics than clinical scores $[47,48]$.

RDT is therefore a tool of choice. It allows the early introduction of an adapted treatment, targeted at a population at risk like ours (risk of RAA). One of the major interests of systematic use of RDT is therefore to have a simple and reproducible diagnostic strategy. Uncertainty is limited there by the existence of a single decision node (negative or positive RDT) [49, 50].

The use of RDT is currently very widespread in many countries of the world, notably in North America and Europe where it occupies a primordial place in the management of angina in children. Current recommendations in most of these countries have benefited from the provision of RDT and tend to limit antibiotic therapy to strep throat only [40, 47, 51]. However, the place of clinical criteria, RDT and bacterial culture of the throat swab differs from country to country (Table 1).

Table 1. Summary and comparison of diagnostic strategies in different recommendations for clinical practice. ESCMID, European Society of Clinical Microbiology and Infectious Diseases; AAP, American Academy of Pediatrics; NICE, National Institute for health and Care Excellence.

\begin{tabular}{llll}
\hline & EuropeESCMID2012 [58] & USAAAP2013 [59] & Royaume-UniNICE2008 [60] \\
\hline Scoreclinique & Non & MacIsaac & Centor \\
TDR & Systématique & Siscore $\geq 2$ & Non \\
Culture & Non & SiTDR- & Non \\
Antibiotique & SiTDR + & SiTDRouculture+ & Siscore $\geq 3$ \\
\hline
\end{tabular}

With us in Morocco as in Tunisia, angina is a very frequent pathology responsible for an excessive pharmaceutical expenditure on antibiotics. The attitude adopted in our countries is to treat all erythematous and erythematatopultacea tonsillitis with antibiotics active on GABHS without recourse to an etiological diagnosis. The frequency of angina and the economic burden caused by often unnecessary antibiotic therapy are arguments in favor of the introduction of RDT in the management of pediatric angina in our country. From a budgetary point of view, the cost of RDT would be lower than the cost of an antibiotic treatment for most RDTs available on the market [52]. It is therefore necessary to draw up recommendations for good practice concerning the management of acute angina in our country. Therefore, it would be wise to recommend carrying out an RDT before any therapeutic decision to limit the use of antibiotics.

No resistance of GABHS to $\beta$-lactams has been described in the literature despite intense use of these molecules in the treatment of angina [36, 47, 53].

In our study, the sensitivity to macrolides (erythromycin, spiramycin and lincomycin) of isolated SBHGA strains demonstrated a strong resistance to erythromycin reaching $69 \%$, with co-resistance reaching $30 \%$ for lincomycin and spiramycin.

Except by reviewing the literature, the rates of resistance to erythromycin are much less important and should sound an alarm bell vis-à-vis the high rates of resistance to macrolides in our country and especially at home in Marrakech linked to a selection pressure too high by self-medication and empirical and not justified prescription of these molecules essentially in the treatment of angina in children. A correlation between resistance to erythromycin and consumption of macrolides has been observed in certain countries such as Italy [54].

Macrolide resistance has been linked to a wide consumption of these antibiotics, and surveillance studies in France have shown that a decrease in the use of macrolides could lead to a decrease in resistance to macrolides [55-57]. Resistance is more frequently linked to young age, which corresponds to a higher prescription in children and a greater risk of cross-contamination than in adults [86]. These results reinforce the recommendations concerning the use of $\beta$ lactams as first line in the treatment of acute angina, and in case of impossibility of taking a bacteriological sample to test the sensitivity of SGA to macrolides before the prescription of this one.

\section{Conclusion}

This study showed a very poor correlation between the clinical criteria and the RDT which did not exceed 33\%. However, we find a very good correlation between the RDT and the bacteriological culture positive for GABHS which was around $80 \%$ with $77 \%$. This testifies to the superiority of RDT compared to the clinical in the detection of angina with GABHS and therefore of a better management and a more reasoned prescription of antibiotics. However, despite the fact that RDT is a great diagnostic aid proven in particular by the different parameters noted by our study, a more sensitive, new rapid molecular $S$. pyogenes tests are emerging to possibly to replace culture, and decrease overtreatment if negative results [61]. Several of these tests are now available, which means they can be performed at the point of care by medical staff, resulting in turnaround times comparable with rapid antigen testing. Although the manufacturers' package inserts claim high sensitivity and specificity compared with culture, there are few data on their performance beyond this. 
Also, these tests are complex tests that require different considerations than rapid antigen testing, so including amplicon contamination? Inhibition/interference? The third concern is the true financial benefit of rapid molecular testing witch is more expensive in place of rapid antigen testing and culture? While waiting to be able to answer these questions in accordance with our socio-economic context, the antigenic RTD constitutes an important contribution in the algotithm of taking care of streptococcal angina in children.

\section{References}

[1] Kline JA, Runge JW. Streptococcal pharyngitis: review of the physiotherapy, diagnosis and treatment. The Journal of emergency medicine 1994; 665-680.

[2] E. MALLET. Etiology, clinical expression of angina. Mdd Mal Infect. 1997; 27: 418-23.

[3] CENTORRM, WHITHERSPOONJM, DALTONHP, BRODYCE, LINKK. The diagnosis of strep throat in adults in the emergency room. Med Decis Making 1981; 1: 239-246.

[4] MCISAACWT, White D, Tannenbaum D, Low DE. A clinical score to reduce unnecessary antibiotic use in patients with sore throat. Canadian medical association journal 1998; 158: 75.83.

[5] MACISAACWJ, Vivel Goel, Teresa To, Low DE. The validity of a score throat in family practice. Canadian medical journal 2000; 163 (7): 811-815.

[6] M Bouskraoui, A. Abid. Acute angina. Service de Pédiatrie A, Hôpital Mère-Enfants, CHU Mohamed VI, Université Cadi Ayyad. Rev Mar Mal Enf 2013; 31: 5-15.

[7] PCIME in developing countries, justification for an integrated strategy. PCIME-OMSWHO/CHS/CAH98./ARev. 1.1999.

[8] BOUROUS M, AIMADEDINE M, AMINE M, OUEDRAOGO V, BOUSKRAOUI M. Assessment of the burden of angina by general practitioners in Settat (Morocco). Médecine et Maladies Infectieuses 2010; 40: 243-244.

[9] Integrated Child Care algorithm according to the Ministry of Health of Morocco Direction of Population 2012 Edition.

[10] S Charachon. Diagnosis and follow-up ORL infections. Le bon usage des examens biologiques. $77 ; 90.98$.

[11] Cardiovascular diseases. Ministry of Health. Available from: http://www.sante.gov.ma/Programmes/mal_cardio/Pages/defa ult.aspx.

[12] BISNOAL. Acute pharyngitis: etiology and diagnosis. Pediatrics 1996; 97 (6pt2): 949-54.

[13] David L. Rheumatic fever, diagnosis and treatment. Archives of Pediatrics. 1998; 5. (6): 681-6.

[14] DENNY FW Jr. A 45 years prespective on the streptococcus and rheumatic fever: the Edward H. Kass lecture in infectious disease history. Clin Inf Dis 1994; 19: 1110-22.

[15] SNELLMANL W, STANGH J, STANGJ M, JOHNSON DR, KAPLAN EL. Duration of positive throat cultures for group A streptococci after initiation of antibiotic therapy. Pediatrics 1993; 91: 1166-70.
[16] OLIVIER C, and the GRAPH group. Acute Rheumatic Fever (ARF) in France: incidence of a first episode during the period 1995-97 in the 5-14 years old children. 38 ${ }^{\text {th }}$ ICAAC 1998. Session 144 L, abstract L90.

[17] KROBER MS, BASS JW, MICHEL SGN. Streptococcal pharyngitis: Placebo-controlled double-blind evaluation of clinical response to penicillin therapy. Jama 1985; 253: 1271-4.

[18] HARRISON F. WOOD, M. D.; ALVANR. FEINSTEIN, M. D.; ANGELOTARANTA, M. D.; JEANNEA. EPSTEIN, M. D.; RITASIMPSON, B. A. Rheumatic fever in children and adolescents. A long term epidemiologic study of subsequent prophylaxis streptococcal infections and clinical sequelae. III. Comparative effectiveness of three prophylaxis regimens in preventing streptococcal infections and rheumatic recurrences. Ann Int Med 1964; 60: 31-46.

[19] DELMAR C. Managing sore throat: a literature review -II- Do antibiotics confer benefit? Med J Austr 1992; 156: 644-9.

[20] MONNETP. What to do in front of an acute strain. Should we treat all angina? Rev Prat. 1965; 15: 971-6.

[21] Statements of American Heart Association-Council in rheumatic fever and congenital heart disease: protection of rheumatic fever patients. JAMA. 1953; 151: 141-3.

[22] SHULMANS. T, GERBERM. A, TANZR R, MARKOVIT ZM. Streptococcal pharyngitis: the case for penicillin therapy. Pediatr Infect Dis J. 1994; 13: 1-7.

[23] SCHWARZR. H., WIENTZENR. L., PEDRERAF, FEROLIEJ, MELLAGW, GUANDOLOVL.-Penicillin V for group A pharyn-gotonsillitis. A randomized trial of seven vs ten days' therapy. JAMA. 1981; 246: 1790-5.

[24] DAJANIA. S, BISNOA. L., CHUNGKJ, DURACKDT, GERBERMA, KAPLANEL et al.-Special report. Prevention of rheumatic fever. A statement for health professionals by the committee on rheumatic fever, endocarditis, Kawazaki disease of the council of cardiovascular disease in the young. Circulation. 1988; 78: 1082-6.

[25] BARONS, BEGUEP. - Antibiotic treatment of angina. Med Mal Infect. 1988; 18: 521-5

[26] PORTIERH. - What's new in the treatment of angina? Lettre Infectiol. 1996; 11: 43-5.

[27] COHEN R., LEVYC, DOIT C, DELAROCQUEF, BOUCHERATM, FITOUSSIF, LANGUEJ, BINGENE: Sixday amoxicillin versus ten day penicillin $\mathrm{V}$ in group $\mathrm{A}$ streptococcal tonsillopharyngitis. Pediatr Infect Dis J. 1996; 15: 678-82.

[28] SHVARTZMANP, TABENKINH. ROSENTZWAIGA, DOLGINOVF.-Treatment of streptococcal pharyngitis with amoxicillin once a day. BMJ. 1993; 306: 1170-2.

[29] Grijalva CG, Nuorti JP, Griffin MR. Antibiotic prescription rates for acute respiratory tract infections in US ambulatory settings. JAMA 2009; 302: 758-66.

[30] Linder JA, Bates DW, Lee GM, Finkelstein JA. Antibiotic treatment of children with sore throat. JAMA 2005; 294: 2315-22.

[31] Allemand H. Inventory of the consumption of antibiotics France. Resistance to Antibiotics: What is at stake today in the city of Nantes? Table of the CNAM, 19mai2010. 
[32] Goossens H, Elseviers Metal. Outpatient antibiotic use in Europeand association with resistance: across-national data base study. Lancet2005; 365: 579-87.

[33] BOUROUSM

AIMADEDINEM,

AMINEM, OUEDRAOGOV, BOUSKRAOUIM. Inventory of the consumption of antibiotics France. Resistance to Antibiotics: What is at stake today in the city of Nantes? Table of the CNAM Médecine et maladies infectieuses 2010; 40: 243-244.

[34] Jérémie COHEN. Diagnostic strategies for childhood pharyngitis from rapid diagnostic test to clinical decision rules. Thesis publicly supported on October 2, 2014.

[35] Buchbinder N, Benzdira A, Belgaid A, Dufour D, Paon J-C, Maurel A et al. Strep throat in pediatric emergencies: performance and impact of a rapid diagnostic test. Original submission. Archive depédiatrie 14 (2007) 1057-1061.

[36] Benouda A, Sibile S, Ziane Y, Elouenass M, Dahani K, Hassani A. Place of Streptococcus pyogenes in tonsillitis in Morocco and current state of antibiotic sensitivity. Pathol Biol 2009; 57: 76-80.

[37] Mezghani Maalej S, Rekik M, Boudaouara M, Jardak N, Turki $\mathrm{S}$, Arous R. et al. Acute angina in children in the Sfax region (Tunisia): epidemiology and interest of the rapid diagnostic test. Médecine et maladie infectieuse 40 (2010) 226-231.

[38] French Agency for the Health Safety of Health Products. Acute Angina. MedMalInfect1999; 29: 227-36.

[39] Turkheim H. General antibiotic therapy in current practice: angina. General method, recommendation and arguments. Arch Pediatr 2003; 10: 260-71.

[40] French agency for the health safety of health products. General antibiotic therapy in common practice in upper respiratory infections in adults and children. 2005 Recommendations.

[41] Gerber Ma, Shulman ST. Rapid diagnosis of pharyngitis caused by group A streptococci. Clin MIcrobiol Rev 2004; 17: 571-80.

[42] Roe M, Kichiyama C, Davidson K, Schaefer L, Todd J. Comparison of biostar Strep A OIA Optical Immune Assay, Abbott Test Pack Plus Strep A, and culture for selective media for diagnosis of group A strptoccocal pharyngitis. J Clin Microbiol 1995; 33: 1551-1.

[43] Cohen R, Chaumette L, Bingen E, et al. The future in angina: rapid diagnostic tests. Med Mal Infect 1997; 27: 424-33.

[44] Committee on infectious deseaces. Group A streptococcal infection. In: Pickering LK, editor. 2000 Red Book. Elk grove village, IL: American Academy of pediatrics 2001. p 526-36.

[45] Webb KH. Does culture confirmation of hight-sensitivity rapid streptococcal test make sense? A medical decision analysis. Pediatrics 1998; 101: E2.

[46] Webb KH, Needham CA, Kurtz SR. Use of a hight-sensitivity rapid strep test without confirmation of negative results: 2 years experience. J Fam Pract 2000; 49: 34-8.

[47] Bisno AL, Gerber MA, Gwaltney Jr. J M, et al. Practice guidelines for the Diagnosis and management of group A streptococcal pharyngitis. Clin Infect Dis 2002; 35: 113-25.

[48] McIsaac WJ, Kellner JD, Aufricht P, et al. Empirical validation of guidelines for the management of pharyngitis in children and adult. JAMA 2004; 291: 1587-95.

[49] Hall MC, Kieke B, Gonzales R, et al. Spectrum bias of a rapid detection test for group A beta-hemolytic streptococcal pharyngitis in a pedratic population. Pedratics 2004; 114: 1826.

[50] Bisno A, Peter GS, Kaplan EL. Diagnosis of strep throat in adults: are clinical criteria really good enought? Clin Infect Dis 2002; 35: 126-9.

[51] Portier H, Peyramond D, Boucot I, Grappin M, Boibieux A, Pribil C. Assessment of the applicability of the consensus on the management of angina in adults. Med Mal Infect 2001; 31: 388-95.

[52] Portier H, Payramond D, Boucot I, Pribil C, Grappin M, Chicoye A, et al. Pharmaco-economic evaluation of the use of rapid diagnostic tests in angina. Med Mal Infect 2001; 31: 506-14.

[53] Jeguirim $H$. Use of a rapid diagnostic test for blood with group A beta-hemolytic streptococcus in pediatric settings. Doctoral thesis in medicine. Faculty of Medicine of Tunis 2006.

[54] Bassetti M, Manno E, Collida A, Ferrando A, Gatti G, Ugolotti E et al. Erythromic in resistance in Streptococcus pyogenes in Italy. Emerg Infect Dis 2000; 6: 180-3.

[55] Fujita K, Murono K, Yoshikawa M, Murai T. Decline of erythromycin resistance of group A streptococci in Japan. Pediatr Infect Dis J 1994; 13: 1075-8.

[56] Seppäla H, Klaukka T, Vuopio-Varkila J, Muotiala A, Helenus $\mathrm{H}$, Lager $\mathrm{K}$, et al. The effect of changes in the consumption of macrolide antibiotics on erythromycin resistance in group A streptococci in Finland. N Engl J Med 1997; 337: 441-6.

[57] Seppäla H, Klaukka T, Lehtonen R, Nenonen E, Huovinen P. The Finnish Study Group for Antimicrobial Resistance. Outpatient use of erythromycin: link to increased erythromycin resistance in group A streptococci. Clin Infect Dis 1995; 21: 1378-85.

[58] Peluchi C, Gregorian L, Galeone C, Esposito S, Huovino P, LittleP et al. Guidelines for the management of acute sore throat. Clin Microbiol Infec2012; 18Suppl1: 1-28.

[59] Hersh AL, Jackson MA, Hichs LA. Principals of judicious antibiotic prescribing for upper respiratory tract infections in pediatrics. Pediatrics 2013; 132: 1146-54.

[60] Nice. Respiratory tract infections-antibiotic prescribing 2008 [updated July 2008 January 2011]. Available from: http://www.nice.org.uk/CG69.

[61] Erin H Graf. Can Rapid Molecular Streptococcus pyogenes Testing Lead to Better Antimicrobial Stewardship for Acute Pharyngitis? The Journal of Applied Laboratory Medicine, Volume 4, Issue 2, 1 September 2019, Pages 140-142. 